\title{
ON NEAR-PERFECT NUMBERS WITH TWO DISTINCT PRIME FACTORS
}

\author{
XIAO-ZHI REN and YONG-GAO CHEN ${ }^{凶}$
}

(Received 29 December 2012; accepted 12 January 2013; first published online 11 March 2013)

\begin{abstract}
Recently, Pollack and Shevelev ['On perfect and near-perfect numbers', J. Number Theory 132 (2012), 3037-3046] introduced the concept of near-perfect numbers. A positive integer $n$ is called near-perfect if it is the sum of all but one of its proper divisors. In this paper, we determine all near-perfect numbers with two distinct prime factors.
\end{abstract}

2010 Mathematics subject classification: primary 11A25; secondary $11 \mathrm{~B} 83$.

Keywords and phrases: perfect number, sum-of-divisors function, near-perfect number.

\section{Introduction}

A positive integer is called a perfect number if it is the sum of all of its proper divisors. It is well known that Euler proved that an even perfect number can be written as $2^{p-1}\left(2^{p}-1\right)$, where both $p$ and $2^{p}-1$ are primes. Primes of the form $2^{p}-1$ are called Mersenne primes. Lenstra, Pomerance, and Wagstaff have conjectured that there are infinitely many Mersenne primes (see the discussion in [1]).

Following Pollack and Shevelev [2], a positive integer $n$ is called a near-perfect number if it is the sum of all but one of its proper divisors. The missing divisor $d$ is called redundant. That is, $n$ is near-perfect with redundant divisor $d$ if and only if $d$ is a proper divisor of $n$ and $\sigma(n)=2 n+d$. Pollack and Shevelev [2] constructed the following three types of near-perfect numbers.

Type 1. $n=2^{t-1}\left(2^{t}-2^{k}-1\right)$, where $2^{t}-2^{k}-1$ is prime, and $2^{k}$ is the redundant divisor.

Type 2. $n=2^{2 p-1}\left(2^{p}-1\right)$, where both $p$ and $2^{p}-1$ are prime numbers, and $2^{p}\left(2^{p}-1\right)$ is the redundant divisor.

Type 3. $n=2^{p-1}\left(2^{p}-1\right)^{2}$, where both $p$ and $2^{p}-1$ are prime numbers, and $2^{p}-1$ is the redundant divisor.

This work was supported by the National Natural Science Foundation of China, Grant No. 11071121.

(c) 2013 Australian Mathematical Publishing Association Inc. 0004-9727/2013 \$16.00 
REMARK 1.1. If $p$ and $2^{p}-1$ are prime numbers, then

$$
n=2^{p}\left(2^{p}-1\right)=2^{p}\left(2^{p+1}-2^{p}-1\right)
$$

is a near-perfect number with redundant divisor $2^{p}$. In this case, $n=2 m$, where $m=2^{p-1}\left(2^{p}-1\right)$ is an even perfect number. Pollack and Shevelev [2, Proposition 3] proved that if $n=2^{j} m$ is a near-perfect number, where $m$ is an even perfect number, then either $n=2^{p}\left(2^{p}-1\right)$ or $n=2^{2 p-1}\left(2^{p}-1\right)$, where both $p$ and $2^{p}-1$ are prime numbers. It is clear that if the Lenstra-Pomerance-Wagstaff conjecture is true, then there are infinitely many near-perfect numbers.

We observe that near-perfect numbers of types 1, 2 and 3 have two distinct prime factors. It is easy to see that 40 is a near-perfect number with redundant divisor 10 , and 40 is not of type 1,2 or 3 .

In this paper, we determine all near-perfect numbers with two distinct prime factors.

THEOREM 1.2. All near-perfect numbers with two distinct prime factors are of types 1 , 2 and 3, together with 40.

Among the first 39 near-perfect numbers which are listed in A181595 in [3], except for the number $40,21(12,20,56,88,104,368,464,992,1504,1888,1952,16256$, $24448,28544,30592,32128,98048,122624,128768,130304,507392)$ are of type 1 ; three $(24,224,15872)$ are of type 2 ; three $(18,196,15376)$ are of type 3 , and $11(234$, $650,3724,5624,9112,11096,13736,17816,77744,174592,396896)$ have three distinct prime factors. D. Johnson has found that $173369889=3^{4} \times 7^{2} \times 11^{2} \times 19^{2}$ the smallest odd near-perfect number and P. Moses has verified that this is the only odd near-perfect number up to $1.4 \times 10^{19}$ (see A181595 in [3]). (From the definition of near-perfect numbers, one may see that all odd near-perfect numbers are squares.)

We pose the following conjecture.

Conjecture 1.3 . For any integer $k \geq 3$, there are only finitely many near-perfect numbers with $k$ distinct prime factors.

\section{Preliminary lemmas}

To prove Theorem 1.2, we first give the following two lemmas.

LEMMA 2.1. If $n=2^{\alpha} q$ is a near-perfect number with redundant divisor $d=2^{s} q$, where $q$ is an odd prime, then either $n=40$ or $n$ is of type 2.

Proof. Since $n=2^{\alpha} q$ is a near-perfect number with redundant divisor $d=2^{s} q$, it follows that $\left(2^{\alpha+1}-1\right)(q+1)=2^{\alpha+1} q+2^{s} q$. That is, $\left(2^{s}+1\right) q=2^{\alpha+1}-1$. This implies that $s \geq 1$.

Let $k$ and $r$ be two integers with $0 \leq r \leq s-1$ such that $\alpha+1=k s+r$. Then

$$
2^{\alpha+1}-1 \equiv 2^{k s+r}-1 \equiv(-1)^{k} 2^{r}-1 \quad\left(\bmod 2^{s}+1\right) .
$$


Since $\left(2^{s}+1\right) q=2^{\alpha+1}-1$, it follows that $2^{s}+1 \mid(-1)^{k} 2^{r}-1$. Thus, by

$$
\left|(-1)^{k} 2^{r}-1\right| \leq 2^{r}+1<2^{s}+1,
$$

we have $(-1)^{k} 2^{r}-1=0$. This implies that $k$ is even and $r=0$. Let $k=2 m$. Thus, by $\left(2^{s}+1\right) q=2^{\alpha+1}-1=2^{2 s m}-1$,

$$
q=\left(2^{s}-1\right) \frac{2^{2 s m}-1}{2^{2 s}-1} .
$$

Since $q$ is an odd prime, it follows that either $2^{s}-1=1$ or $2^{2 s m}-1=2^{2 s}-1$. Thus, either $s=1$ or $m=1$.

If $s=1$, then $\alpha+1=2 m$. Thus $\left(2^{s}+1\right) q=2^{\alpha+1}-1$ becomes $3 q=\left(2^{m}-1\right) \times$ $\left(2^{m}+1\right)$. Since $\left(2^{m}-1,2^{m}+1\right)=1$ and $q \geq 3$, it follows that $m=2, q=5$ and $\alpha=3$. Thus $n=40$.

If $m=1$, then $q=2^{s}-1$ is an odd prime and $\alpha=2 s-1$. Hence $n=2^{2 s-1}\left(2^{s}-1\right)$ is of type 2 .

This completes the proof of Lemma 2.1.

LEMMA 2.2. Let $q$ be an odd prime, $\alpha$ and $\beta$ positive integers with $\beta \geq 2$. If $n=2^{\alpha} q^{\beta}$ is a near-perfect number with redundant divisor $d$, then $q^{\beta} \nmid d$.

Proof. Suppose that $d=2^{s} q^{\beta}$ with $0 \leq s \leq \alpha-1$. We will derive a contradiction.

Since $\sigma(n)=2 n+d$, it follows that

$$
\left(2^{\alpha+1}-1\right)\left(1+q+\cdots+q^{\beta}\right)=\left(2^{\alpha+1}+2^{s}\right) q^{\beta} .
$$

If $\beta$ is even, then $1+q+\cdots+q^{\beta}$ is odd. Thus $s=0$. Since

$$
\left(2^{\alpha+1}-1,2^{\alpha+1}+1\right)=1, \quad\left(1+q+\cdots+q^{\beta}, q^{\beta}\right)=1,
$$

it follows from (2.1) that

$$
2^{\alpha+1}-1=q^{\beta}, \quad 1+q+\cdots+q^{\beta}=2^{\alpha+1}+1 .
$$

Thus

$$
2^{\alpha+1}+1=2^{\alpha+1}-1+2=q^{\beta}+2<1+q+\cdots+q^{\beta}=2^{\alpha+1}+1,
$$

a contradiction.

Now we assume that $\beta$ is odd. Then the left-hand side of (2.1) is even. Thus $s \geq 1$. From (2.1),

$$
2^{s} \mid 1+q+\cdots+q^{\beta}
$$

and

$$
\left(2^{\alpha+1}-1\right)\left(1+q+\cdots+q^{\beta-1}\right)=\left(2^{s}+1\right) q^{\beta} .
$$

By $(2.3)$ and $\left(q^{\beta}, 1+q+\cdots+q^{\beta-1}\right)=1$,

$$
1+q+\cdots+q^{\beta-1} \mid 2^{s}+1
$$

We distinguish two cases according to the value of $\beta$. 
Case 1: $\beta \equiv 1(\bmod 4)$. Since

$$
1+q+\cdots+q^{\beta}=(1+q)\left(1+q^{2}+q^{4}+\cdots+q^{\beta-1}\right)
$$

and

$$
1+q^{2}+q^{4}+\cdots+q^{\beta-1} \equiv \frac{\beta+1}{2} \not \equiv 0 \quad(\bmod 2),
$$

it follows from (2.2) that $2^{s} \mid 1+q$. Thus, by $\beta \geq 3$, we have $2^{s}+1 \leq q+2<1+q+$ $\cdots+q^{\beta-1}$, a contradiction with (2.4).

Case 2: $\beta \equiv 3(\bmod 4)$. Since

$$
1+q+\cdots+q^{\beta}=\left(1+q^{2}\right)\left(1+q+q^{4}+q^{5}+\cdots+q^{\beta-3}+q^{\beta-2}\right)
$$

and $4 \nmid 1+q^{2}$, it follows from (2.2) that $2^{s-1} \mid 1+q+q^{4}+q^{5}+\cdots+q^{\beta-3}+q^{\beta-2}$. Thus, by (2.4) and $\beta \geq 3$,

$$
q 2^{s-1} \leq q\left(1+q+q^{4}+q^{5}+\cdots+q^{\beta-3}+q^{\beta-2}\right) \leq 2^{s}+1-1=2^{s},
$$

a contradiction with $q$ being an odd prime. This completes the proof of Lemma 2.2.

\section{Proof of Theorem 1.2}

Let $n=p^{\alpha} q^{\beta}$ be a near-perfect number with redundant divisor $d$, where $p$ and $q$ are two primes with $p<q$, and $\alpha, \beta$ are two positive integers. Then $\sigma(n)=2 n+d$. If $p \geq 3$, then

$$
\sigma(n)=\frac{p^{\alpha+1}-1}{p-1} \frac{q^{\beta+1}-1}{q-1}<\frac{p^{\alpha+1}}{p-1} \frac{q^{\beta+1}}{q-1}=n \frac{p}{p-1} \frac{q}{q-1} \leq \frac{3 \times 5}{2 \times 4} n<2 n .
$$

Hence $p=2$. Let $d=2^{s} q^{t}$ with $0 \leq s \leq \alpha$ and $0 \leq t \leq \beta$. Thus, $\sigma(n)=2 n+d$ becomes

$$
\left(2^{\alpha+1}-1\right)\left(1+q+\cdots+q^{\beta}\right)=2^{\alpha+1} q^{\beta}+2^{s} q^{t} .
$$

We distinguish three cases according to the value of $\beta$.

Case 1: $\beta=1$. Then $t \in\{0,1\}$. If $t=0$, then, by (3.1), $q=2^{\alpha+1}-2^{s}-1$ is an odd prime and $d=2^{s}$. Thus $n=2^{\alpha}\left(2^{\alpha+1}-2^{s}-1\right)$ is of type 1 . If $t=1$, then $n=2^{\alpha} q$ is a near-perfect number with redundant divisor $d=2^{s} q$. By Lemma 2.1, either $n=40$ or $n$ is of type 2.

Case 2: $\quad \beta=2$. By Lemma 2.2, $q^{2} \nmid d$. Thus $t \in\{0,1\} . \quad$ By (3.1), $s=0 . \quad$ If $t=0$, then, by (3.1), $\left(2^{\alpha+1}-q\right)(1+q)=2$, a contradiction. Hence $t=1$. By (3.1), $q=2^{\alpha+1}-1$ is an odd prime. Therefore, $n=2^{\alpha}\left(2^{\alpha+1}-1\right)^{2}$ is of type 3 . 
Case 3: $\beta \geq 3$. By Lemma 2.2, $0 \leq t \leq \beta-1$. The equality (3.1) can be rewritten as

$$
\left(2^{\alpha+1}-q\right)\left(1+q+\cdots+q^{\beta-1}\right)=1+2^{s} q^{t} .
$$

If $q<2^{\alpha}$, then the left-hand side of (3.2) is more than $2^{\alpha}\left(1+q+\cdots+q^{\beta-1}\right)$. Noting that $\beta \geq 3$, the right-hand side of (3.2) does not exceed $1+2^{\alpha} q^{\beta-1}<2^{\alpha}(1+q+\cdots+$ $q^{\beta-1}$ ), a contradiction. Hence $q \geq 2^{\alpha}$. Since the left-hand side of (3.2) is at least

$$
1+q+\cdots+q^{\beta-1}>1+q^{2} \geq 1+2^{\alpha} q \geq 1+2^{s} q
$$

we have $t \geq 2$. By (3.1), $q \mid 2^{\alpha+1}-1$, a contradiction with $q \geq 2^{\alpha}$.

This completes the proof of Theorem 1.2.

\section{Acknowledgements}

We are grateful to the referee for helpful comments and the editor for suggesting that we give a list of some known near-perfect numbers.

\section{References}

[1] R. Crandall and C. Pomerance, Prime Numbers: A Computational Perspective, 2nd edn (Springer, New York, 2005).

[2] P. Pollack and V. Shevelev, 'On perfect and near-perfect numbers', J. Number Theory 132 (2012), 3037-3046.

[3] N. J. Sloane, 'The online encyclopedia of integer sequences', available at http://oeis.org/.

XIAO-ZHI REN, School of Mathematical Sciences and Institute of Mathematics, Nanjing Normal University, Nanjing 210023, PR China

YONG-GAO CHEN, School of Mathematical Sciences and Institute of Mathematics, Nanjing Normal University, Nanjing 210023, PR China

e-mail: ygchen@njnu.edu.cn 